*Preprint version. Scheduled for publication in the 2014 Charleston Conference Proceedings.

\author{
The Buck Stops Here: \\ Assessing the Value of E-book Subscriptions at the Columbia University Libraries \\ Melissa J. Goertzen, Columbia University, mjg2227@columbia.edu \\ Krystie Klahn, Columbia University, kk2926@columbia.edu
}

\begin{abstract}
:
Over the past four years Columbia University Libraries (CUL) has seen exponential growth in electronic book (e-book) purchasing. These purchases have not only increased the depth and breadth of the collection, but they have also created new opportunities for remote learning and instant information access. In turn, this new push for purchasing electronic has created new demands in assessment to understand the true benefit of these resources, most notably in regards to annual e-book subscriptions.

In 2013, a new position aimed at developing an e-book strategy for CUL was devised. Shortly thereafter, a position was created in the Science and Engineering Library Division (SEL) that focused on assessment. These two positions fall in line with CUL's mission to support research and learning through evidence based decision making.

This paper explains an assessment methodology used within the library system at CUL to evaluate the cost-benefit of e-book subscriptions. By appraising several databases, we were able to analyze cost and usage to determine the actual value of these resources. The findings yielded a savings of approximately $\$ 60,000$ for the 2015 fiscal year. This is an ongoing initiative that will help us document the e-book landscape and build data sets that will inform collection development decisions.
\end{abstract}

\title{
Part 1: Introduction
}

The dawning of the Digital Age revolutionized the way information and knowledge are created, produced, and disseminated in the academic community. The rapid integration of technologies with research, teaching, and learning activities has changed both information and access needs of user communities. A new reliance on electronic content coupled with the pressure of reduced stack space for print collections are factors that contributed to a new focus on electronic book (ebooks) acquisition initiatives in academic libraries. Librarians now face the challenge of assessing and evaluating this new format in regards to the value it offers to users.

Columbia University Libraries (CUL) is one of the top five academic research library systems in North America and serves a community of over 3,750 faculty members and 26,000 full-time students at the Morningside Campus and Medical Center. The collections are housed across twenty-one campus libraries and include over twelve million volumes, 160,000 current journals and serials, and an extensive collection of manuscripts, rare books, microforms, maps, and audiovisual materials. In 2004, CUL began purchasing e-books in an experimental capacity. Due to positive reception by faculty and students, CUL began expanding e-book collections to support research, teaching, and learning activities across campus. Currently, CUL provides 
access to more than two million e-book titles and expenditures comprise 25 per cent of the total book budget.

As the e-book collection continues to grow, CUL is developing a unique strategy and vision for e-book collection development, programs, and initiatives. To achieve this goal, the Collection Development Department launched the E-Book Program Development Study in 2013. This assessment project centers on the collection of essential data to drive the development of policies related to e-book acquisition, discovery, and access. In 2014, the Science and Engineering Library Division (SEL) created the position of Collection Assessment and Analysis Librarian in order to promote regular and standardized assessment of existing collections.

In 2014, 96 active e-book subscriptions were identified at CUL. The objective of this paper is to describe an assessment methodology used to evaluated the cost-benefit of these e-book subscriptions across the entire library system, and then within SEL. By appraising several databases, we were able to analyze the cost, usage, and value of these resources. This work involved conducting cost analysis, examining usage trends, and conducting title overlap analysis. The study was guided by the following four principles outlined in the CUL/IS Strategic Plan $2010-2013$ :

1. User-focused design;

2. Data-driven decision making;

3. Continuous assessment of results;

4. Flexible and adaptive response to user needs (Columbia University Libraries, 2010, p. 8).

The assessment method described in this paper was applied on two separate occasions in the Collection Development Department and SEL between November 2013 - May 2014. In both cases, the method provided actionable results and contributed to the standardization of e-book assessment and negotiation strategies across campus. The findings yielded a savings of approximately $\$ 60,000$ for the 2015 fiscal year. Since savings are tied to subscription packages, they will carry forward into coming years. In addition, both studies resulted in discussions with vendors regarding price negotiation and platform performance. Finally, we gained a deeper understanding of how resources can be allocated to best support the needs of user communities. The ongoing evaluation of e-book subscriptions will allow for evidence based decision making and assist in the continued documentation of the e-book landscape at CUL.

\section{Part 2: E-Book Program Development Study}

The E-Book Program Development Study is an ambitious, two year assessment project aimed at gathering essential data to drive the development of policies related to e-book development programs. It aligns with CUL's mission to support the development and delivery of high-quality services that facilitate research, teaching, and learning across campus and within the wider scholarly community. The results will provide a set of recommendations and policies for internal and external stakeholders as they collaborate on the development and implementation of e-book projects and programs. 
The objective at the heart of the E-Book Program Development Study is to develop a strategy and vision for e-book programs and collections at CUL. Essentially, the set of recommendations that result from study findings will create a bridge between the current landscape and CUL's vision for future e-book initiatives on campus. A large part of this work involves the development of methodologies that examine how e-book resources are allocated, evaluate current subscriptions and packages, examine usage trends, and build collaborative relationships with vendors. The data that was gathered while developing these methodologies will be used to inform recommendations and policy statements regarding e-book collection development and management on campus.

The reality that the e-book landscape is constantly evolving was factored into decisions regarding the overarching assessment framework guiding this study. The research design was created so that it can be replicated regardless of how e-books evolve in the coming years. Because the design is flexible and adaptive in nature, it promotes continued assessment, evaluation, and strategic planning as a regular component of e-book programs.

As part of this study, a large-scale cost analysis project was completed between November 2013 - March 2014. The goal of the project was to collect quantitative data that will inform e-book collection development policies in regards to fund allocation and preferred business models. While the study examined both e-book subscriptions and purchases, for the purposes of this paper, the discussion will be limited in scope to e-book subscriptions.

After discussions with the Director of Collection Development, and the Head of Electronic Resources Management, it was determined that e-books are most often purchased on the EO fund code. To collect financial data for all e-book subscriptions, a Voyager (CUL's integrated library system) query was run for all library funds ending in EO. After running the cumulative query, we created a base list of all e-book subscriptions at CUL. Given the enormous size of this data set and the time restrictions placed in the study, data collection was limited to subscriptions that had fund activity during the 2013 fiscal year (FY2013). This limited the base list to 96 ebook subscriptions. Next, subscription fees in FY2013 were totaled, and calculations were made to identify the top 70 per cent (bulk) and bottom 30 per cent (tail) of purchases within the budget. Statistical analysis was also conducted to determine the total, average, median, high, and low costs.

To examine usage trends, the top four subscriptions (ranked by cost) were selected and corresponding title lists were collected from the Continuing \& Electronic Resources Management (CERM) Division. At the same time, the corresponding BR2 COUNTER report was pulled from the vendor website. At this point, we encountered an unexpected challenge; in several cases, multiple collections from the same vendor are purchased as separate items on the EO fund code. However, there is no apparent way to filter COUNTER reports by collection. At this point, we considered analyzing the data by vendor instead of by collection, but decided that this method would skew results because of the discrepancies in cost, size, and use. Instead, we filtered the data for a second time by matching the 2013 title lists with COUNTER report data. Based on these results, we calculated the number of titles loaned, number of loans, percentage of titles without use after purchase, the average cost of an e-book, and cost per use (see Table 1). Please note that the results were calculated using confidential data. For the purposes of this 
paper, the numbers were changed and percentages are not exact, but they reflect the trends discovered in the actual study findings.

\begin{tabular}{|l|c|c|c|c|}
\cline { 2 - 5 } \multicolumn{1}{c|}{} & Subscription A & Subscription B & Subscription C & Subscription D \\
\hline 2013 Cost & $\$ 50,000.00$ & $\$ 20,000.00$ & $\$ 15,000.00$ & $\$ 60,000.00$ \\
\hline No. of titles & 80,000 & 6,000 & 125 & 11,000 \\
\hline No. of titles loaned & 34,000 & 2,100 & 90 & 1,600 \\
\hline No. of loans & $2,500,00$ & 11,900 & 22,00 & 6,500 \\
\hline $\begin{array}{l}\text { \% of titles without } \\
\text { use after purchase }\end{array}$ & $62 \%$ & $65 \%$ & $25 \%$ & $85 \%$ \\
\hline $\begin{array}{l}\text { Average cost of } \\
\text { e-book }\end{array}$ & $\$ 0.60$ & $\$ 3.00$ & $\$ 140.00$ & $\$ 5.00$ \\
\hline Cost per use & $\$ 0.20$ & $\$ 0.20$ & $\$ 0.75$ & $\$ 9.00$ \\
\hline
\end{tabular}

Table 1. Recalculated results of the e-book subscription cost and usage analysis.

After analyzing the cost and usage data of the top e-book subscriptions, it was determined that the cost per use of Subscription D was high ( $\$ 9.00$ per use) compared to Subscription A $(\$ 0.20$ per use), Subscription B ( $\$ 0.20$ per use), and Subscription C ( $\$ 0.75$ per use).

The results were presented to the E-Resource Usage Data Working Group (ERUDWG) at CUL. The consensus was to conduct a second analysis of Subscription D based on the following criteria: evaluation of content, overlap analysis, and interface analysis. The results indicated that Subscription D contained a large number of outdated technical manuals (96 per cent published before 2011), a high number of titles available through other platforms, and incomplete multivolume sets.

Next, a team of librarians from SEL examined the title list and identified 394 high use titles (more than 20 page views). Of this subset, she discovered that 196 titles (49.75 per cent) are available through other platforms at CUL. Then, she searched GOBI for the remaining 198 high use titles and discovered that the vast majority are available for individual purchase. Based on this analysis, it was determined that Subscription D does not contain a significant amount of unique content. A decision was made to cancel the subscription.

One unexpected outcome of this analysis was the opportunity to speak with the Vice President and a team of sales representatives managing Subscription D on three separate occasions. The company requested feedback from CUL regarding how to improve the platform interface and content, and were provided with study findings. After a series of negotiations Subscription D was renewed for one year at an 80 per cent discount, resulting in a cost savings of $\$ 50,000$. At the end of FY2014, Subscription D will be assessed using the same methodology to determine if it will be renewed the following year. At this time, we are also investigating a number of marketing initiatives to promote content and will monitor usage rates to determine if there are noticeable changes over the next twelve months.

\section{Part 3: E-Books in the Science and Engineering Library}


The Science and Engineering Library Division (SEL) at CUL was once comprised of eight different libraries. Over the past few years there have been a series of library closures, with the most recent being the closure of the Engineering Library in June of 2014, bringing the total to four libraries. The closures have physically reduced library space and have forced the consolidation of a majority of print books to off-site storage. In addition, these closures coupled with the strong acceptance of electronic content by faculty and students have made SEL the perfect subject for e-book development. There is now a preference policy to purchase electronically when available. This in turn has led to the desire to learn how cost effective electronic collections are and if new models of purchasing would be beneficial.

In recent years, the SEL has moved away from subject specialist roles to focus on a functional role structure. This structure will allow our positions to evolve in a seamless manner regardless of how the landscape at SEL changes in the coming years. While there are still purchasing and liaison responsibilities related to specific subjects, librarian roles focus on division wide functions. For example, in May of 2014 a new position was created to focus on collection assessment and analysis of materials for the entire science division. This new position allows for the designation of much needed time towards the evaluation of our large collection of electronic and print content.

With regards to electronic content, increased cost trends that are inconsistent with library budgets provided the catalyst for initiating the new role. There needed to be a systematic and concentrated effort on assessment to ensure collections warranted purchase and were seen as valuable to the collection. Thus this position allows for the justification of purchases as well as cancellations. Moreover, continued analysis is beneficial in maintaining quality curation of collections. It can highlight under-utilized content as well as reaffirm valuable resources. Indepth looks at electronic content can even point to discovery or access issues, thus addressing silent threats.

The first assessment project for this role analyzed several subscription based e-book packages from one vendor (Vendor A). Vendor A provides access to over forty e-book subject collections. In 2014, CUL subscribed to eleven of these collections. Over the past few years each collection has seen a four to seven per cent cost increase annually, and overall usage of these e-books dipped in 2013. It was also noted that Vendor A reserves the right to drop content from collections without adjusting the price. Instead, they add content which is not always comparable. Before renewing these subscriptions, we needed to find out if each collection was still worthwhile for our library. We decided to conduct a cost analysis to prove or disprove benefit.

We were able to acquire usage data from COUNTER compliant BR2 reports posted on Vendor A's admin site, which aligns with our preference mandate to collect standardized data sets. When we compared usage statistics against fund data gathered from Voyager, we found that the eleven collections seemed like a good deal with a low cost per use $(\$ 2.24)$, which is in line with comparable electronic subscriptions at CUL. However, we also needed to account for the fact that CUL does not subscribe to the eleven collections from Vendor A in one lump sum, but rather subscribes to each on an individual basis. 
During assessment, we also discovered a 27 per cent overlap of titles across the eleven collections we subscribe to from Vendor A. This per cent overlap increased between each package and some overlap reached as high as 66 per cent. Upon further investigation, we also found that this content overlapped with titles available through other e-book platforms at CUL. This discovery prompted a deeper analysis of content available through Vendor A, and we discovered a long tail of use with a handful of titles receiving heavy use and the majority receiving low (in many cases zero) uses. In total, our patrons used approximately 25 per cent of collection content. Although this is a common trend in e-book subscriptions, we flagged the collections for further investigation nonetheless.

We also noticed that the cost per use for each collection ranged from $\$ .38$ to $\$ 11.21$. While some collections were well worth the purchase price, others seemed to be far too high a cost. After breaking down the purchases, there were three collections highlighted as questionable (see Table 2 and 3$)$.

\begin{tabular}{|l|r|r|r|r|}
\cline { 3 - 5 } & \multicolumn{3}{|c|}{} & \multicolumn{3}{c|}{ Questionable Packages } \\
\cline { 2 - 5 } & Vendor A Subscription & Package F & Package J & Package K \\
\hline \# of Titles in Database & 9161 & 717 & 713 & 238 \\
\hline \# of Titles Overlapped & 2444 & 471 & 241 & 121 \\
\hline \% of Overlap & $27 \%$ & $66 \%$ & $34 \%$ & $51 \%$ \\
\hline
\end{tabular}

Table 2. Results of the overlap analysis for Vendor A (FY2013).

\begin{tabular}{|c|c|c|c|c|c|c|}
\hline & & & \multicolumn{4}{|c|}{ Questionable Packages } \\
\hline & \multicolumn{2}{|c|}{ Vendor A Subscription } & Package F & \begin{tabular}{|l|} 
Package J \\
\end{tabular} & Pac & rage $\mathrm{K}$ \\
\hline Cost & $\$$ & $50,000.00$ & $\$ 6,000.00$ & $\$ 3,000.00$ & $\$$ & $2,500.00$ \\
\hline Unique Usage & & 22324 & 841 & 351 & & 223 \\
\hline$\%$ Used & & $25 \%$ & $27 \%$ & $11 \%$ & & $21 \%$ \\
\hline Cost Per Use & $\$$ & 2.24 & 7.13 & 8.55 & $\$$ & 11.21 \\
\hline
\end{tabular}

Table 3. Recalculated results of the cost and usage analysis for Vendor A based on BR2 COUNTER data (FY2013).

These findings were presented to our colleagues in SEL as well as ERUDWG for discussion. Open conversations with these groups helped us to further analyze the subscriptions and compile feedback that was presented to Vendor A. As a group, we decided to cancel the two lowest used collections and we negotiated a flat percentage increase for two years with the remaining nine collections. With the flat price increase, we will see a savings of $\$ 10,000$ annually and believe these collections will be sustainable and cost effective going forward.

\section{Part 4: Conclusion: Library as Advocate and Negotiator}

Our findings demonstrate that standardized usage reporting in the form of COUNTER reports are beneficial to the assessment of collections. The reports allow "the usage of online information products and services to be measured in a credible, consistent and compatible way using vendorgenerated data" (ProjectCOUNTER, 2012, p. 1). Information such as title, publication year, and 
unique identifiers like DOI help librarians understand the genuine usage patterns at their institutions. When vendors are compliant with COUNTER it makes assessment more manageable and meaningful by standardizing what is considered use. Therefore, we are no longer comparing apples to oranges but rather apples to apples.

The decisions that were made using this assessment methodology will not only affect our budgets but also the content in our collections. We learned that it is important to present data and findings to library divisions as well as committees or working groups to weigh options and provide feedback. Different viewpoints can point out issues that may have been initially excluded from assessment plans. In addition, discussing findings can increase awareness of initiatives, such as marketing collections to promote discovery, which may result in greater buyin from stakeholders, thus increasing the value of e-book collections.

The idea that the term "librarian" is not synonymous with "negotiator" must be changed. We need be our own advocates, make informed decisions, and demand change if change is needed. Large price increases that are inconsistent with subscription use should not be tolerated. Assessment projects such as the one described in this paper, prove the true value of resources and can be effectively used to negotiate acceptable price tags based on use.

The reality that the e-book landscape is constantly evolving was factored into decisions regarding the overarching assessment framework. The research design was created so that it can be replicated regardless of how e-books evolve in the coming years. Because the design is flexible and adaptive in nature, it promotes continued assessment, evaluation, and strategic planning as a regular component of e-book programs. This study proves that this methodology can be used on varying collection sizes and provides actionable results.

In closing, systematic and routine assessment of collections is important in determining the true value of resources for our user community. It is imperative to make informed decisions when negotiating renewals rather than passing the "buck." We hope that the experiences and findings outlined through this paper will help others implement an assessment program at their institution.

\section{References}

Columbia University Libraries. (2010, January). Strategic Plan, 2010 - 2013. Retrieved from https://culis.columbia.edu/content/ dam/staffweb/admin/strategicplanning/ 20102013/CULIS_Strategic_Plan_2010-20131.pdf

ProjectCOUNTER (2012, April). The COUNTER Code of Practice for e-Resources: Release 4. Retrieved from http://www.projectcounter.org/r4/COPR4.pdf

\section{Acknowledgements}

We would like to thank Daisy Alcron at Columbia University for her assistance with data collection from Voyager, Serial Solutions, and COUNTER usage reports. 